\title{
A novel compact leaky wave antenna incorporating the dual-periodical loading
}

\author{
Chang Lu ${ }^{1}$, Hsien-Shun Wu ${ }^{1 \text { a) }}$, and Ching-Kuang Tzuang ${ }^{1,2}$ \\ ${ }^{1}$ School of Electronic Information Engineering, Tianjin University, \\ Tianjin 300072, People's Republic of China \\ ${ }^{2}$ THZ Microelectronics Science and Technologies Co., Ltd., \\ Beijing 100176, People's Republic of China
}

a)seanwu@ieee.org

\begin{abstract}
This paper presents a novel leaky wave antenna (LWA) loaded by periodical open stubs and holes. The complex propagation constants of the first higher order mode of the periodically loaded microstrip, so-called the EH1 mode, are extracted by the field eigenvalue approach, leading to control the radiation characteristics by changing the loading conditions. By doubling the length of open stub, the operating frequency of the LWA can be shifted $8.9 \%$ toward to the lower frequency. The comparison of the dispersion curves between the loaded and conventional microstrips shows $20 \%$ width reduction on synthesizing the EH1 mode. Two $9 \mathrm{GHz}$ and $10 \mathrm{GHz}$ practical prototypes are designed based on the proposed methodology and fabricated by using the multilayer printed circuit board (PCB) technology. The measured return-losses of two LWA prototypes are lower than $10 \mathrm{~dB}$. The measured antenna gains of two LWA are $5.37 \mathrm{dBi}$ and $6.89 \mathrm{dBi}$. The maximum gains are $6.05 \mathrm{dBi}$ and $7.43 \mathrm{dBi}$ at 9.2 and $10.1 \mathrm{GHz}$. The beam scanning angles are 7 degrees and 4 degrees when the frequency is increased $0.2 \mathrm{GHz}$.
\end{abstract}

Keywords: periodical loading, leaky wave antenna, microstrip, print-circuit-board

Classification: Electromagnetic theory

\section{References}

[1] A. A. Oliner and K. S. Lee: "The nature of the leakage from higher modes on microstrip line,” IEEE MTT-S Int. Microw. Symp. Dig. (1986) 57 (DOI: 10. 1109/MWSYM.1986.1132108).

[2] A. A. Oliner: "Leakage from higher modes on microstrip line with application to antennas," Radio Sci. 22 (1987) 907 (DOI: 10.1029/RS022i006p00907).

[3] C. Luxey and J. M. Laheurte: "Simple design of dual-beam leaky-wave antennas in microstrips," IEE Proc., Microw. Antennas Propag. 144 (1997) 397 (DOI: 10.1049/ip-map:19971407).

[4] J. L. Gómez-Tornero, et al.: "Design of tapered leaky-wave antennas in hybrid waveguide-planar technology for millimeter waveband applications," IEEE Trans. Antennas Propag. 53 (2005) 2563 (DOI: 10.1109/TAP.2005.850741). 
employing the coupled-mode approach," IEEE Trans. Microw. Theory Techn. 49 (2001) 629 (DOI: 10.1109/22.915436).

[6] Y.-D. Lin, et al.: "Analysis and design of feeding structures for microstrip leaky wave antenna," IEEE Trans. Microw. Theory Techn. 44 (1996) 1540 (DOI: 10. $1109 / 22.536602)$.

[7] C.-N. Hu and C.-K. C. Tzuang: "Injection-locked coupled microstrip leakymode antenna array," IEE Proc., Microw. Antennas Propag. 147 (2000) 364 (DOI: 10.1049/ip-map:20000599).

[8] J.-W. Wu, et al.: "A compact wideband leaky-wave antenna with etched slot elements and tapered structure," IEEE Trans. Antennas Propag. 58 (2010) 2176 (DOI: 10.1109/TAP.2010.2048847).

[9] C.-K. Wu, et al.: "Compressed-width leaky EH1 mode PBG antenna," IEEE Microw. Wireless Compon. Lett. 13 (2003) 343 (DOI: 10.1109/LMWC.2003. 815692).

[10] A. Niembro-Martín, et al.: "Slow-wave substrate integrated waveguide," IEEE Trans. Microw. Theory Techn. 62 (2014) 1625 (DOI: 10.1109/TMTT.2014. 2328974).

[11] X. R. Li, et al.: "Anomalous dispersion characteristics of periodic substrate integrated waveguides from microwave to terahertz," IEEE Trans. Microw. Theory Techn. 63 (2015) 2142 (DOI: 10.1109/TMTT.2015.2431265).

\section{Introduction}

The first higher-order mode of the conventional microstrip line, so-called the leaky mode was theoretically explored by A. A. Oliner [1]. The leaky wave antennas, designed based on the principle of the leaky mode, had been extensively demonstrated the advantages on the high-gain, high-efficient, frequency-scanning characteristics $[2,3,4,5,6]$. The conventional LWA, implemented by using the standard microstrip line, has two structural parameters to control the frequency range of the leaky mode, including the permittivity of the dielectric substrate and the width of the microstrip [7]. Recently, the rectangular slots are integrated into the signal and the ground plane of the tapered LWA to disturb the current distributions on the metal surfaces for making the electromagnetic radiation at lower frequency without increasing the width of the LWA [8]. By comparing the conventional LWA design, the EME (electric-magnetic-electric) microstrip integrates the photonic bandgap (PBG) structure on the signal trace to perturb the modal current of the first higher order mode (EH1), effectively realizing the LWA with $20 \%$ reduction on the line width [9]. This paper uses dual-periodical loading to control the EH1 mode of the microstrip, making a novel leaky wave antenna. The dual-periodical loading, including the open stubs and etched holes, are applied to realize the signal trace of the microstrip. The rigorous field theoretical eigenvalue approach is reported in Section 2 to extract the complex propagation constants of the EH1 mode of the loaded microstrip, demonstrating how the proposed dual loading affecting the characteristic of the leaky mode. The theoretical normalized phase constants, defined by the ratio of $\beta$ to $\beta_{0}$ in this paper, lead to design the LWA prototypes. Two antenna prototypes are fabricated by the standard four-layer print circuit board process. Section 3 reports the measured results, experimentally proving the meas- 
ured return-losses lower than $10 \mathrm{~dB}$. The maximum gains are $7.43 \mathrm{dBi}$ and $6.05 \mathrm{dBi}$ at 10.1 and $9.2 \mathrm{GHz}$. The beam scanning angles are 7 degrees and 4 degrees when the frequency is increased $0.2 \mathrm{GHz}$. Section 4 concludes the paper.

\section{Microstrip with periodical loading}

The proposed microstrip line loaded with the periodical open stubs and the etched hole is constructed by the unit cell shows the caption of Fig. 1(a). The unit cell, defined by the four structural parameters, usually has a size about fifty times smaller than the wavelength in the free space at the operating frequency of the microstrip. The structural parameters of the unit cell include the periodicity $(\mathrm{P})$, the width of the etched hole $\left(\mathrm{W}_{\mathrm{h}}\right)$, the diameter $\left(\mathrm{D}_{\mathrm{p}}\right)$ and the length of the open stub $\left(\mathrm{H}_{\mathrm{p}}\right)$. If $\mathrm{W}_{\mathrm{h}}$ and $\mathrm{H}_{\mathrm{p}}$ are set to zero, the proposed structure can become the conventional microstrip.

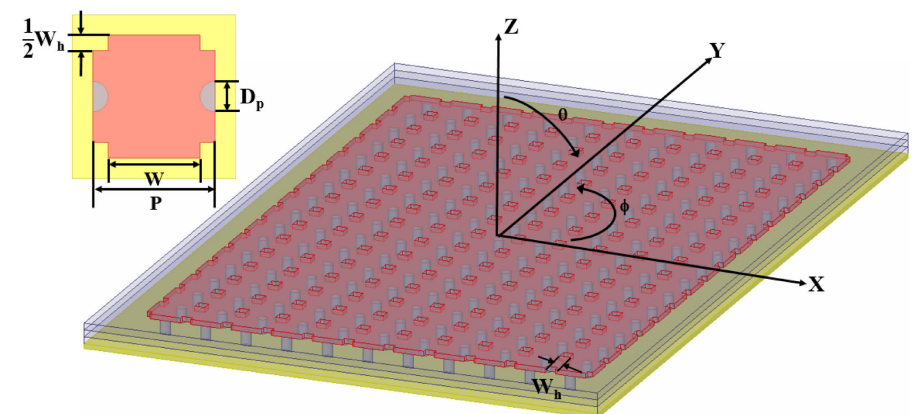

(a)

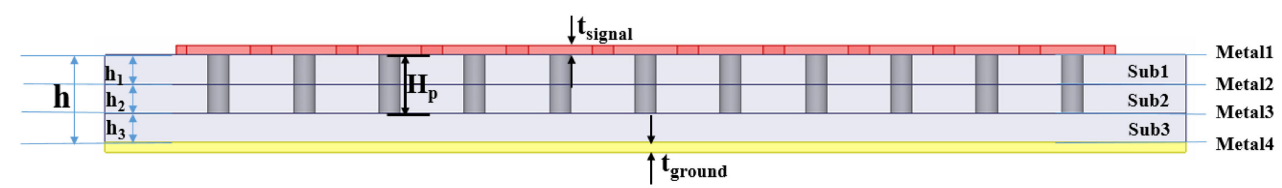

(b)

Fig. 1. Microstrip with dual-periodical loading in the multilayer laminated substrates: (a) Three-dimensional view of the structure, (b) Cross-section view of the structure. Structural parameters: $\mathrm{W}=450 \mu \mathrm{m}, \mathrm{P}=600 \mu \mathrm{m}, \mathrm{D}_{\mathrm{p}}=150 \mu \mathrm{m}, \mathrm{t}_{\text {signal }}=$ $\mathrm{t}_{\text {ground }}=16 \mu \mathrm{m}, \mathrm{h}_{1}=\mathrm{h}_{2}=\mathrm{h}_{3}=100 \mu \mathrm{m}$, permittivity $\left(\varepsilon_{\mathrm{r}}\right)$ of the substrate: 4.7 , dielectric loss: 0.008 .

The open stub was applied to affect the phase constants of the substrate integrated waveguide [10]. This paper uses the periodical open stub and the etched holes to control the propagation constants of the first higher order mode (EH1) of the microstrip. As shown in Fig. 1, the proposed microstrip consists of $\mathrm{M}$ unit cells in the transverse direction and $\mathrm{N}$ unit cells in the longitudinal direction. To extract the complex propagation constants of the proposed two-dimensional periodic microstrip, the numerical procedures based on the hybrid eigenvalue approach are conducted [11]. Before the extractions, the $\mathrm{M}$ by $\mathrm{N}$ multiport scattering parameters of the loaded microstrip are calculated by using the commercial software, HFSS ANSYSTM. Then by solving the eigenvalue formulation in Eq. (1) with the periodic Floquet-Bloch boundary condition, the complex eigenvalue, $\gamma$, are obtained. 


$$
\left[\begin{array}{l}
\mathrm{v}_{1} \\
\mathrm{i}_{1}
\end{array}\right]=\left[\begin{array}{ll}
\mathrm{A} & \mathrm{B} \\
\mathrm{C} & \mathrm{D}
\end{array}\right]\left[\begin{array}{l}
\mathrm{v}_{2} \\
\mathrm{i}_{2}
\end{array}\right]=\mathrm{e}^{\mathrm{N} \cdot \cdot \cdot \mathrm{p}} \cdot \mathrm{I} \cdot\left[\begin{array}{l}
\mathrm{v}_{2} \\
\mathrm{i}_{2}
\end{array}\right]
$$

Fig. 2(a) shows the extracted result of the normalized phase constant $\left(\beta / \beta_{0}\right)$ of the dominant microstrip mode (EH0) of the proposed microstrip with different periodical loadings. The $\mathrm{M}$ and $\mathrm{N}$ are set as 11 and 4, respectively. The normalized phase constant is defined as the slow wave factor (SWF) in this paper. The black curve, which $\mathrm{W}_{\mathrm{h}}$, and $\mathrm{H}_{\mathrm{p}}$ are set to zero, represents the SWF of the conventional microstrip. The red curve, indicating the SWF of the microstrip only loaded by the etch holes, is nearly identical to the curve of the conventional microstrip from $2 \mathrm{GHz}$ to $20 \mathrm{GHz}$. The comparisons between the black and red curves show that the etch holes produce a little effects on the microstrip mode. The blue and green curves, which indicate the microstrip loaded by the open stubs of 100 and $200 \mathrm{um}$, represent the SWF of 2.4 and 2.7 , showing $9.1 \%$ and $22.7 \%$ increasing from the conventional microstrip. The open stub, whose size is 150 times smaller than the wavelength at $20 \mathrm{GHz}$, can artificially elevate the SWF of the microstrip without changing the material parameters. The numerical data in Fig. 2(a) theoretically demonstrate the capability of the proposed structure on changing the guiding characteristics of the microstrip in the dominant mode (EH0). This paper also extends the modal analyses on the first higher order mode (EH1) of the proposed structure with different loading.

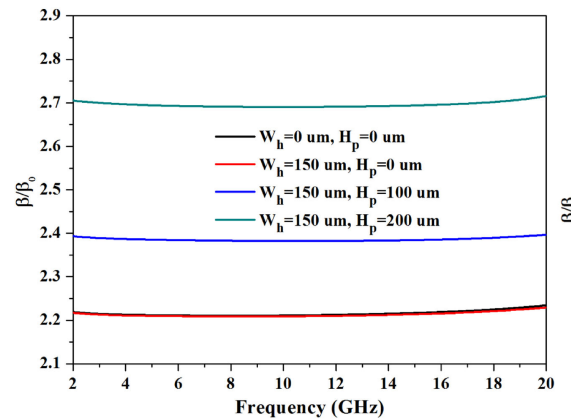

(a)

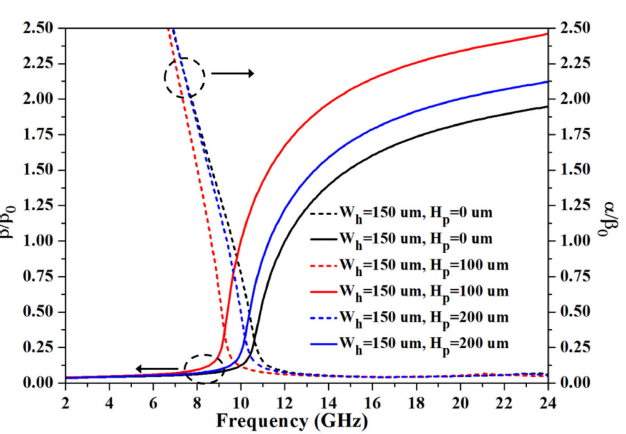

(b)

Fig. 2. The extracted complex propagation constants of the microstrip with different periodical loading: (a) Dominant mode (EH0) of the microstrip with different dual-periodical loading, (b) First higher order mode (EH1) of the microstrip with different dualperiodical loading.

Fig. 2(b) shows the normalized propagation constants of the EH1 mode. The dashed lines represent the normalized attenuation constant equal to $\alpha / \beta_{0}$, and the solid lines represent the normalized phase constant equal to $\beta / \beta_{0}$. The cutoff frequency of the EH1 mode is defined by the intersecting frequency between the curves of the normalized phase and the attenuation constants. The black curve, representing the results of the $\mathrm{H}_{\mathrm{p}}$ equal to zero, shows that the periodical etched holes make the cutoff frequency of the EH1 mode at $10.6 \mathrm{GHz}$. In all the numerical analyses, the width of the proposed microstrip, consisting of 11 unit cells in the transverse direction, is kept as a constant of $6600 \mathrm{um}$. The comparisons in Fig. 2(b) show that the cutoff frequency of EH1 mode can be shifted from $10.6 \mathrm{GHz}$ to 
9.2 GHz by increasing the length of the open stub from $0 \mu \mathrm{m}$ to $200 \mu \mathrm{m}$. Furthermore, Fig. 3 shows the comparison of the dispersion curves of the EH1 mode between the proposed and conventional microstrip. The green curve, which is copied from the blue curve in Fig. 2(b), represents the cutoff frequency of EH1 mode at $10.1 \mathrm{GHz}$. The blue curve, which is the extracted result of the conventional microstrip with a width of $7480 \mathrm{um}$, shows a nearly identical EH1 mode cutoff frequency at $10.1 \mathrm{GHz}$. The comparison between green and blue curves shows an $11.8 \%$ width reduction on synthesizing the EH1 mode. Similarly, the black curve represents the cutoff frequency of the conventional microstrip with 8250 um wide at 9.2 GHz. By doubling the length of the open stub $\left(\mathrm{H}_{\mathrm{p}}\right)$, the width miniaturization ratio can be increased from $11.8 \%$ to $20.0 \%$. The comparisons in Fig. 3 theoretically predict the feasibility of the proposed periodical loading on the leaky wave antenna (LWA) miniaturization. In the next section, the antenna prototypes, which are designed based on the proposed structure, are fabricated and tested to experimentally prove the concept.

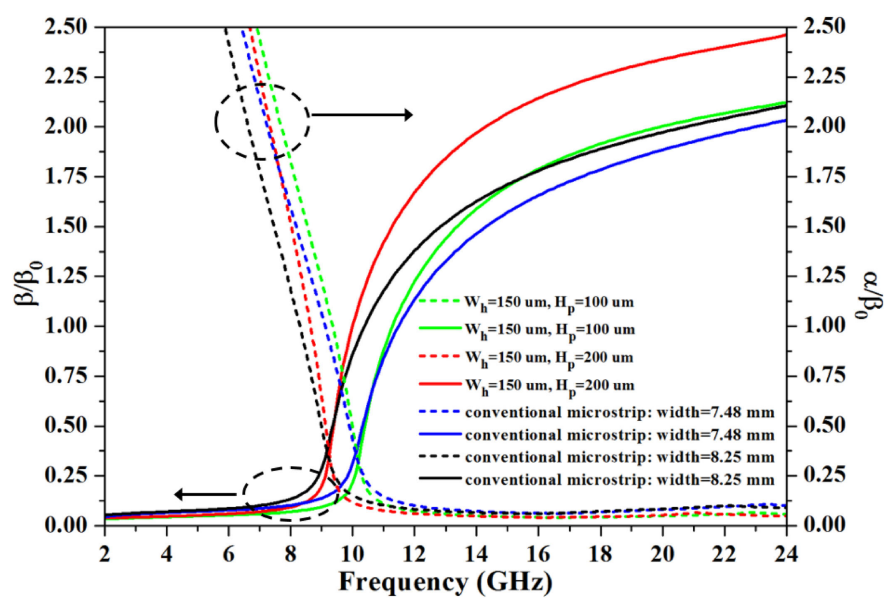

Fig. 3. The dispersion curves of the EH1 mode of the conventional microstrip lines and the microstrip with the dual periodical loading. The width of the loaded microstrip is set as $6600 \mathrm{um}$.

\section{Laminated prototype and experiments}

Using the extracted complex propagation constants represented by the blue and red curves in Fig. 2(b), two leaky wave antennas (LWA) with dual-periodical loading are designed. The designs are conducted by using the identical structural parameters of the unit cell except for the length of open stub. As shown in Fig. 1, the length open stub, denoted by $\mathrm{H}_{\mathrm{p}}$ is set as 100 and $200 \mathrm{um}$, respectively. The standard four-layer print circuit board (PCB) process, whose substrate parameters are identical to those of in Fig. 1, is applied to implement the open stubs and etched holes. The different lengths of the open stubs are realized by using the embedded blind vias process with difference metal layer. When $H_{p}$ is equal to $100 \mathrm{um}$, the blind vias are created from metal 1 to metal 2 layer. When $\mathrm{H}_{\mathrm{p}}$ is set as $200 \mathrm{um}$, the vias are created from metal 1 to metal 3 layer. Fig. 4 shows the photographic of the LWA with 200 um-long open stubs. The prototype consists of 11 cells in the transverse direction, and has 260 cells in the longitudinal direction, corresponding to five-time of the guiding wavelength at $10 \mathrm{GHz}$. The single-end to differential 
matching network, shown on the left-hand side of the Fig. 4, makes the impedance transformation from the $50-\Omega$ system to the microstrip with dual-periodical loading.

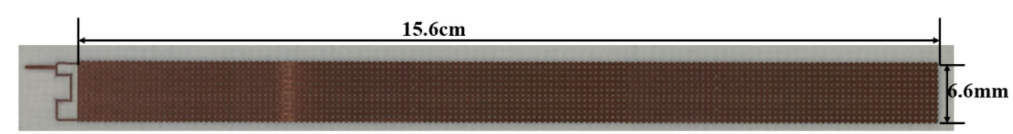

Fig. 4. Photographic of the proposed leaky wave antenna (LWA) with 200 um long of the open stub. The effective area of the antenna is $15.6 \mathrm{~cm} \times 6.6 \mathrm{~cm}$.

The two-port vector network analyzer, E8363A, Keysight ${ }^{\mathrm{TM}}$, is applied to characterize the input return loss of the two prototypes after conducting the oneport short-open-load (SOL) calibration. Fig. 5 shows the comparison between the simulated and the measured results of two prototypes. All the measured results include the effects of the standard $3.5 \mathrm{~mm}$ SMA connectors. The LWA with the open stub of $100 \mathrm{um}$ is designed at $10 \mathrm{GHz}$, and the measured return-loss less than $10 \mathrm{~dB}$ from $9.9 \mathrm{GHz}$ to $10.35 \mathrm{GHz}$. The antenna with $200 \mathrm{um}$ long open stub is designed at $9 \mathrm{GHz}$. The curves in Fig. 5(b) show the return loss less than $10 \mathrm{~dB}$ from $8.95 \mathrm{GHz}$ to $9.45 \mathrm{GHz}$.

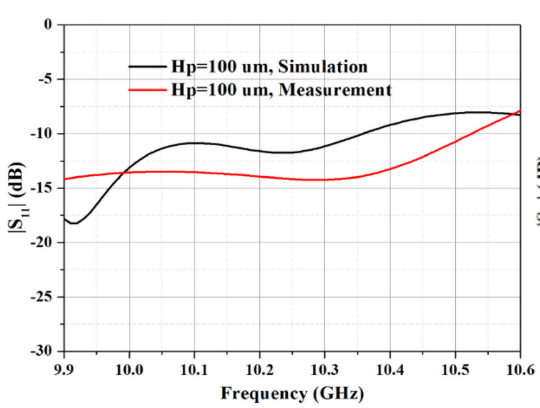

(a)

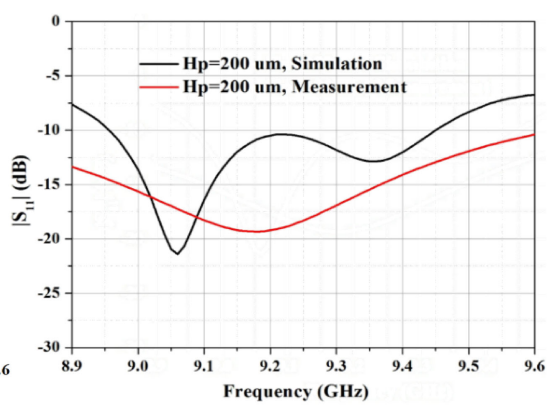

(b)

Fig. 5. The simulated and the measured input return loss of the proposed LWA prototypes in $50-\Omega$ system: (a) $\mathrm{H}_{\mathrm{p}}=100 \mathrm{um}$, (b) $\mathrm{H}_{\mathrm{p}}=200 \mathrm{um}$.

The measurements of the far-field radiation patterns are conducted by using the $40 \mathrm{GHz}$ RF chamber made by WavePro ${ }^{\mathrm{TM}}$. The gain calibrations are performed by using the X-band standard gain horn before the radiation patterns characterizations.

Fig. 6 shows both simulated and measured far-field radiation patterns of LWAs at $\phi$ equal to $90^{\circ}$ in H-plane. In Fig. 6(a), the antenna gain of the LWA with open stubs of $100 \mathrm{um}$ is about $6.89 \mathrm{dBi}$ from $10.1 \mathrm{GHz}$ to $10.3 \mathrm{GHz}$. The maximum gain is $7.43 \mathrm{dBi}$ at $10.1 \mathrm{GHz}$. The gain difference between the simulation and the measurement is less than $0.16 \mathrm{dBi}$, revealing excellent agreement. In average, the backward radiations are kept $5.5 \mathrm{~dB}$ lower than the main beams between $\theta=0$ and $\theta=90$ degrees, indicating most electromagnetic energy radiating along the longitudinal direction of the LWA. Additionally, in Fig. 6(b), the LWA prototype loaded with $200 \mathrm{um}$ open stubs has a measured gain of $5.37 \mathrm{dBi}$ and has a maximum gain of $6.04 \mathrm{dBi}$ at $9.2 \mathrm{GHz}$. Both simulations and the experiments confirm the main beam at $38^{\circ}, 41^{\circ}, 42^{\circ}$ from the broadside of the antenna when the input frequency is increased from $9.2 \mathrm{GHz}$ to $9.4 \mathrm{GHz}$. 


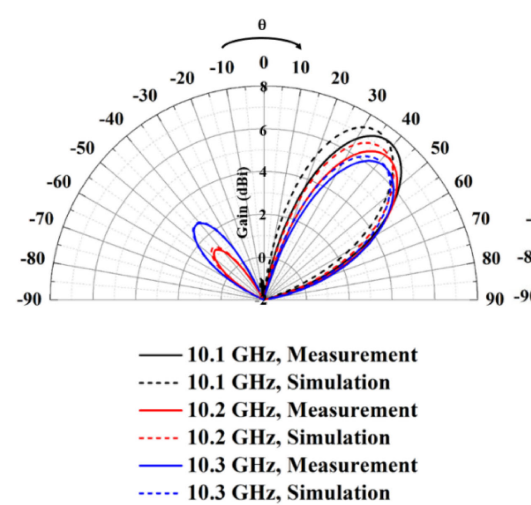

(a)

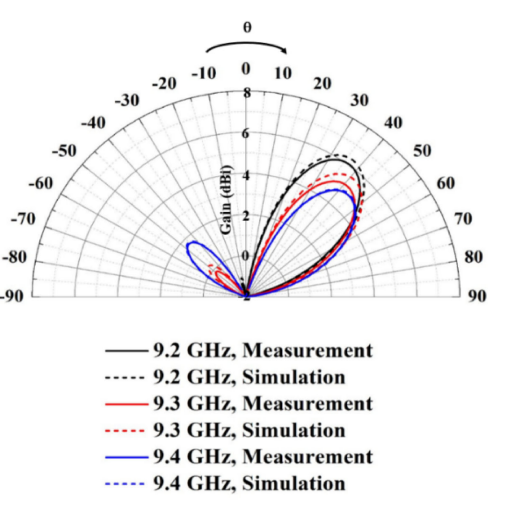

(b)

Fig. 6. H-plane far-field radiations patterns at $\phi=90^{\circ}$ : (a) $H_{p}=$ 100 um, (b) $\mathrm{H}_{\mathrm{p}}=200 \mathrm{um}$.

Table I summarizes the miniaturization rate, the bandwidth, and the antenna gain of two prototypes. The miniaturization rate is equal to the antenna width reduction reported in Fig. 3. The radiation bandwidth is defined by the frequencies corresponding to the normalized phase constant $\left(\beta / \beta_{0}\right)$ equal to 0.3 and 0.8 in Fig. 2(b). The average gains are calculated from the measured data reported in Fig. 6. By doubling the length of the open stub, the bandwidth of the LWA is slightly decreased from $0.7 \mathrm{GHz}$ to $0.6 \mathrm{GHz}$. The radiation gain is reduced about $1.5 \mathrm{dBi}$. The proposed dual-periodical loading can achieve $20 \%$ miniaturization rate and maintain the bandwidth for designing LWA antenna.

Table I. Performances of the proposed LWA with dual periodical loading

\begin{tabular}{c|c|c}
\hline & $\mathrm{H}_{\mathrm{p}}=100 \mathrm{um}$ & $\mathrm{H}_{\mathrm{p}}=200 \mathrm{um}$ \\
\hline Miniaturization Rate & $11.8 \%$ & $20.0 \%$ \\
\hline Bandwidth $(\mathrm{GHz})$ & $10.1 \mathrm{GHz}-10.8 \mathrm{GHz}$ & $9.1 \mathrm{GHz}-9.7 \mathrm{GHz}$ \\
\hline Average Gain $(\mathrm{dBi})$ & 6.896 & 5.366 \\
\hline
\end{tabular}

\section{Conclusion}

A novel leaky wave antenna (LWA) periodically loaded by the open stub and the etched hole is proposed. The extracted complex propagation constants theoretically confirm the proposed dual-periodical loading can effectively make the cutoff frequency of EH1 mode lower 13.2\%. Two prototypes, designed and fabricated based on the different length of the loaded stubs, are experimentally characterized. The measured results, having excellent agreements with the simulations and the calculation, experimentally demonstrate a $20 \%$ miniaturization rate for the LWA design.

\section{Acknowledgments}

This work was supported in part by the National Natural Science Foundation of China under Grant 61271068, and the Global Expert Program in China. 